\title{
New travelling wave solutions for fractional regularized long-wave equation and fractional coupled Nizhnik-Novikov-Veselov equation
}

\author{
Özkan Güner* \\ Department of International Trade, Faculty of Economics and Administrative Sciences, \\ Çankırı Karatekin University, Çankırı, Turkey \\ ozkanguner@karatekin.edu.tr
}

\section{ARTICLE INFO}

\section{Article History:}

Received 15 November 2016

Accepted 16 June 2017

Available 23 October 2017

Keywords:

Exact solution

Ansatz method

$\left(G^{\prime} / G\right)$-expansion method

Fractional regularized long-wave

equation

Fractional coupled Nizhnik-Novikov-

Veselov equation

AMS Classification 2010

$34 A 08,35 R 11,83 C 15$

\section{ABSTRACT}

In this paper, solitary-wave ansatz and the $\left(G^{\prime} / G\right)$-expansion methods have been used to obtain exact solutions of the fractional regularized long-wave (RLW) and coupled Nizhnik-Novikov-Veselov (NNV) equation. As a result, three types of exact analytical solutions such as rational function solutions, trigonometric function solutions, hyperbolic function solutions are formally derived from these equations. Proposed methods are more powerful and can be applied to other fractional differential equations arising in mathematical physics.

\section{Introduction}

Fractional differential equations (FDEs) are the generalized form of classical differential equations of integer order. Researchers especially in applied mathematician and physicist became highly interested in obtaining exact solutions for nonlinear FDEs in recent decades. Nonlinear FDEs are frequently used to describe many problems of physical phenomena that may arise in various fields such as biology, physics, chemistry, engineering, heat transfer, applied mathematics, control theory, mechanics, signal processing, seismic wave analysis, finance, and many other fractional dynamical systems [1-3].

In the past several decades, new exact solutions may help to find new phenomena. So, variety of powerful analytical and numerical methods for solving differential equations of fractional order have been suggested such as the adomian decomposition method, the homotopy perturbation method, the variational iteration method, the finite difference method, the differential transform method, homotopy perturbation method, the homotopy analysis method, the sub-equation method, the first integral method, the $\left(G^{\prime} / G\right)$ expansion method, the modified trial equation method, the functional variable method, the expfunction method, the simplest equation method, the exponential rational function method, ansatz method and others [4-31].

To solve mathematical problems, the transforms are an important methods. A variety of useful transforms for solving different problems appeared in the literature, such as the traveling wave transform, the Fourier transform and the others [32-41]. Recently, Li and He [42] suggested a fractional complex transform to convert FDEs into ordinary differential equations (ODEs).

*Corresponding Author 
There are different kinds of fractional derivative operators. The most famous one is the Caputo definition that the function should be differentiable [43]. Recently, Jumarie derived definitions for the fractional derivative called modified Riemann-Liouville, which are suitable for continuous and non-differentiable functions. The order $\alpha$ of Jumarie's derivative is defined by [44]:

$$
\begin{aligned}
& D_{w}^{\alpha} f(w)= \\
& \left\{\begin{array}{c}
\frac{1}{\Gamma(1-\alpha)} \frac{d}{d w} \int_{0}^{w} \frac{(f(\theta)-f(0))}{(w-\theta)^{\alpha}} d \theta, 0<\alpha<1 \\
\left(f^{(\rho)}(w)\right)^{(\alpha-\rho)}, \rho \leq \alpha<\rho+1, \rho \geq 1 .
\end{array}\right.
\end{aligned}
$$

Some properties of the fractional modified RL derivative are [45]

$$
\begin{gathered}
D_{w}^{\alpha} w^{r}=\frac{\Gamma(1+r)}{\Gamma(1+r-\alpha)} w^{r-\alpha}, \\
D_{w}^{\alpha}(c)=0, \\
D_{w}^{\alpha}\{a f(w)+b g(w)\}=a D_{w}^{\alpha} f(w)+b D_{w}^{\alpha} g(w),
\end{gathered}
$$

where $a, b$ and $c$ are constants.

We take into consideration the following general nonlinear FDE of the type

$$
H\left(u, D_{t}^{\alpha} u, D_{x}^{\beta} u, D_{t}^{2 \alpha} u, D_{t}^{\alpha} D_{x}^{\beta} u, D_{x}^{2 \beta} u, \ldots\right)=0,
$$

where $0<\alpha, \beta<1, H$ is a polynomial of $u, u$ is an unknown function and $D^{\alpha}$ partial fractional derivatives of $u$.

The traveling wave variable

$$
\begin{gathered}
u(x, t)=U(\theta), \\
\theta=\frac{\varepsilon x^{\alpha}}{\Gamma(1+\alpha)}-\frac{\tau t^{\alpha}}{\Gamma(1+\alpha)},
\end{gathered}
$$

where $\tau \neq 0$ and $\varepsilon \neq 0$ are constants. Applying the fractional chain rule

$$
\begin{aligned}
& D_{t}^{\alpha} u=\sigma_{t} \frac{d U}{d \xi} D_{t}^{\alpha} \theta \\
& D_{x}^{\alpha} u=\sigma_{x} \frac{d U}{d \xi} D_{x}^{\alpha} \theta
\end{aligned}
$$

where $\sigma_{t}$ and $\sigma_{x}$ are called the sigma indexes $[46,28]$ and we can choose $\sigma_{t}=\sigma_{x}=L$, where $L$ is a constant.

When we substitute, (6) with (2) and (7) into (5), we can get Eq.(5) in the following NODE;

$$
\Psi\left(U, U^{\prime}, U^{\prime \prime}, U^{\prime \prime \prime}, \ldots, U^{(n)}, \ldots\right)=0
$$

where $U^{(n)}$ is the $n^{\text {th }}$ derivative of $U$ with respect to $\theta$.

\section{Description of the ansatz method for solving FDEs}

For bright solitons, the starting hypothesis is in the form $[47,48]$

$$
u(x, t)=A \operatorname{sech}^{p} \theta
$$

and

$$
\theta=\frac{k x^{\alpha}}{\Gamma(1+\alpha)}-\frac{c t^{\alpha}}{\Gamma(1+\alpha)}
$$

where $A, k$ and $c$ are nonzero constants. From the ansatz given above with two equalities, it is possible to obtain necessary derivatives. Then, the obtained derivatives are substituted in the Eq.(5) and we collect all terms with the same order of necessary terms. Then by equating each coefficient of the resulting polynomial to zero, we obtain a set of algebraic equations for; $A, k$ and $c$. Finally solving the system of equations we can get exact solution of Eq.(5) [49-52].

\subsection{Applications of the proposed method}

Example 1: The space-time fractional RLW equation has the form [53]

$$
D_{t}^{\alpha} u+v D_{x}^{\alpha} u+a u D_{x}^{\alpha} u-\tau D_{t}^{\alpha} D_{x}^{2 \alpha} u=0,
$$

where $\alpha$ describing the order of the fractional derivatives $0<\alpha \leq 1$ and $v, a$ and $\tau$ are all constants that describe the behavior of the undular bore [54]. The RLW equation is modeled to govern a large number of physical phenomena such as nonlinear transverse waves in shallow water, ion acoustic and magneto hydrodynamic waves in plasma and phonon packets in nonlinear crystals. Eq.(11) was first put forward as a model for small amplitude long waves on the surface of water in channel by Peregine [55], and later by Benjamin et al. [56]. This equation is considered as an alternative to the KdV equation. Abdel-Salam and Hassan solved Eq.(11) by the fractional auxiliary sub-equation expansion method [53]. AbdelSalam and Yousif, have obtained abundant types of exact analytical solutions including generalized trigonometric and hyperbolic functions solutions of this equation with the fractional Riccati expansion method in [57]. Analytical solutions of fractional RLW equation is very low. When $\alpha=1$, equation (11) is called the RLW equation. Conversely, many researchers focus on numerical 
methods to obtain approximate solutions of RLW equation. For example, Esen and Kutluay solved the equation by a lumped Galerkin method in [58]. Dag et al. have applied least square quadratic B-spline and cubic B-spline finite element method to obtain new analytical solutions of RLW equation in $[59,60]$. Saka et al. [61,62] solved this equation by quintic B-spline collocation and Bspline collocation algorithms methods. In [63], the variational iteration method successfully applied to finding the solution of the RLW equation by Yusufoglu and Bekir.

In order to solve Eq.(11), we use the traveling wave transformation

$$
\begin{gathered}
u(x, t)=U(\theta), \\
\theta=\frac{k x^{\alpha}}{\Gamma(1+\alpha)}-\frac{c t^{\alpha}}{\Gamma(1+\alpha)},
\end{gathered}
$$

where $k \neq 0$ and $c \neq 0$ are constants. When we substitute (12) with (2) and (6) into (11) and by integrating once and setting the constants of integration to be zero, the Eq. (11) can carry to an ODE

$$
(k v-c) U+\frac{a k}{2} U^{2}+\tau c k^{2} L^{2} U^{\prime \prime}=0,
$$

where $U^{\prime}=\frac{d U}{d \theta}$. The solitary wave ansatz for the bright soliton solution, the hypothesis is (9) and (10). From (9) and (10), it is possible to get

$$
\frac{d^{2} U(\theta)}{d \theta^{2}}=A p^{2} \operatorname{sech}^{p} \theta-A p(p+1) \operatorname{sech}^{p+2} \theta,
$$

and

$$
U^{2}(\theta)=A^{2} \operatorname{sech}^{2 p} \theta
$$

Thus, substituting the ansatz (14) and (15) into Eq.(13), yields to

$$
\begin{aligned}
& (k v-c) A \operatorname{sech}^{p} \theta+\frac{a k}{2} A^{2} \operatorname{sech}^{2 p} \theta \\
& +\tau c k^{2} L^{2} A p^{2} \operatorname{sech}^{p} \theta \\
& -\tau c k^{2} L^{2} A p(p+1) \operatorname{sech}^{p+2} \theta=0 .
\end{aligned}
$$

Now, from (16), equating the exponents $p+2$ and $2 p$ leads to

$$
p=2 .
$$

From (16), setting the coefficients of $\operatorname{sech}^{p+2} \theta$ and $\operatorname{sech}^{2 p} \theta$ terms to zero, we get

$$
\frac{a k}{2} A^{2}-\tau c k^{2} L^{2} A p(p+1)=0,
$$

by use (17) and after some calculations, we have

$$
A=\frac{12 \tau k c L^{2}}{a}, a \neq 0 .
$$

We find, from setting the coefficients of $\operatorname{sech}^{p} \theta$ terms in Eq.(16) to zero

$$
(k v-c) A+\tau c k^{2} L^{2} A p^{2}=0,
$$

also we obtain

$$
c=\frac{v k}{1-4 \tau k^{2} L^{2}} .
$$

From (21) it is important to note that

$$
4 \tau k^{2} L^{2} \neq 1
$$

Thus finally, bright soliton solution of (11) is given by:

$$
\begin{aligned}
u(x, t)= & \frac{12 \tau k c L^{2}}{a} \times \\
& \operatorname{sech}^{2}\left(\frac{k x^{\alpha}}{\Gamma(1+\alpha)}-\frac{v k t^{\alpha}}{\left(1-4 \tau k^{2} L^{2}\right) \Gamma(1+\alpha)}\right) .
\end{aligned}
$$

Example 2: Secondly, we consider the following the space-time fractional coupled NizhnikNovikov-Veselov (NNV) equation [64]

$$
\begin{aligned}
& D_{t}^{\alpha} u-A D_{x}^{3 \alpha} u-B D_{y}^{3 \alpha} u+3 A u D_{x}^{\alpha} v \\
& \quad+3 A v D_{x}^{\alpha} u+3 B u D_{y}^{\alpha} w+3 B w D_{y}^{\alpha} u=0, \\
& D_{x}^{\alpha} u-D_{y}^{\alpha} v=0, \\
& D_{y}^{\alpha} u-D_{x}^{\alpha} w=0,
\end{aligned}
$$

where $0<\alpha \leq 1, A$ and $B$ are given constants satisfying $A+B \neq 0$, and $u, v$ and $w$ are the functions of $(x, y, t)$. Yan has found three types of travelling wave solutions of equation (24) by using the fractional sub-equation method [64]. The $(2+1)$ dimensional NNV equation is an isotropic extension of the well-known (1+1)-dimensional $\mathrm{KdV}$ equation. In recent years, NNV equation have been studied several areas of physics including condense matter physics, optics, fluid mechanics, and plasma physics when $\alpha \rightarrow 1$ [65-67]. Darvishi et al. have applied exp-function method to obtain exact traveling wave solutions of classical NNV equation in [68]. Deng solved the equation by use of the extended hyperbolic function method in 
[69]. In [70], Wazwaz et al. have investigated the bright soliton solutions with wave ansatz method.

For our goal, we present the following transformation

$$
\begin{aligned}
u(x, y, t) & =U(\theta), \theta=\frac{k x^{\alpha}}{\Gamma(1+\alpha)}+\frac{m y^{\alpha}}{\Gamma(1+\alpha)}-\frac{n t^{\alpha}}{\Gamma(1+\alpha)}, \\
v(x, y, t) & =V(\theta), \theta=\frac{k x^{\alpha}}{\Gamma(1+\alpha)}+\frac{m y^{\alpha}}{\Gamma(1+\alpha)}-\frac{n t^{\alpha}}{\Gamma(1+\alpha)}, \\
w(x, y, t) & =W(\theta), \theta=\frac{k x^{\alpha}}{\Gamma(1+\alpha)}+\frac{m y^{\alpha}}{\Gamma(1+\alpha)}-\frac{n t^{\alpha}}{\Gamma(1+\alpha)},
\end{aligned}
$$

where $k \neq 0, m \neq 0$ and $n \neq 0$ are constants.

Then by using of Eq. (25) with (2) and (7), Eq.(24) can be turned into an ODEs and by integrating once and setting the constants of integration to be zero, we obtain

$$
\begin{aligned}
& \left(A k^{3} L^{2}+B m^{3} L^{2}\right) U^{\prime \prime}-3 k A(U V) \\
& \quad-3 m B(U W)+n U=0, \\
& k U-m V=0, \\
& m U-k W=0,
\end{aligned}
$$

where $U^{\prime}=\frac{d U}{d \theta}$ and $V^{\prime}=\frac{d V}{d \theta}$. In order the start off with the solution hypothesis, the following ansatsz is assumed

$$
u(x, y, t)=\lambda_{1} \operatorname{sech}^{p} \theta
$$

and

$$
v(x, y, t)=\lambda_{2} \operatorname{sech}^{s} \theta,
$$

and

$$
w(x, y, t)=\lambda_{3} \operatorname{sech}^{r} \theta
$$

where

$$
\theta=\frac{k x^{\alpha}}{\Gamma(1+\alpha)}+\frac{m y^{\alpha}}{\Gamma(1+\alpha)}-\frac{n t^{\alpha}}{\Gamma(1+\alpha)} .
$$

Here in (27)-(30), $\lambda_{1}, \lambda_{2}, \lambda_{3}, k$ and $m$ are the free parameters of the solitons and $n$ is the velocity of the soliton. The exponents $p, s$ and $r$ are unknown values will be find later. Now, from (27)-(29) and (30) it is possible to obtain

$$
\begin{aligned}
& \left(A k^{3} L^{2}+B m^{3} L^{2}\right) \lambda_{1} p^{2} \operatorname{sech}^{p} \theta \\
& -\left(A k^{3} L^{2}+B m^{3} L^{2}\right) \lambda_{1} p(p+1) \operatorname{sech}^{p+2} \theta \\
& -3 k A \lambda_{1} \lambda_{2} \operatorname{sech}^{p+s} \theta-3 m B \lambda_{1} \lambda_{3} \operatorname{sech}^{p+r} \theta \\
& +n \lambda_{1} \operatorname{sech}^{p} \theta=0
\end{aligned}
$$

and

$$
k \lambda_{1} \operatorname{sech}^{p} \theta-m \lambda_{2} \operatorname{sech}^{s} \theta=0,
$$

and

$$
m \lambda_{1} \operatorname{sech}^{p} \theta-k \lambda_{3} \operatorname{sech}^{r} \theta=0 .
$$

Now from (32) and (33) equating the exponents of $\operatorname{sech} \theta$ functions we have $p=s=r$. In (32) we obtain,

$$
\lambda_{2}=\frac{k \lambda_{1}}{m}
$$

Similarly in (34) that gives

$$
\lambda_{3}=\frac{m \lambda_{1}}{k} .
$$

Now, equating the exponents of $\operatorname{sech}^{p+2} \theta$ or $\operatorname{sech}^{p+s} \theta$ and $\operatorname{sech}^{p+r} \theta$ functions in (31) with $p=s=r$, one gets

$$
p+2=p+s=p+r,
$$

so that

$$
p=s=r=2 .
$$

Setting the coefficients of $\operatorname{sech}^{p+2} \theta$ in (31), to zero gives

$$
\begin{array}{r}
\left(A k^{3} L^{2}+B m^{3} L^{2}\right) \lambda_{1} p(p+1)+3 k A \lambda_{1} \lambda_{2} \\
+3 m B \lambda_{1} \lambda_{3}=0,
\end{array}
$$

using Eqs. (34), (35), $p=2$ and some calculations

$$
\lambda_{1}=-2 k m L^{2} .
$$

Again from (31), setting the coefficients of $\operatorname{sech}^{p} \theta$ terms to zero one obtains,

$$
\left(A k^{3} L^{2}+B m^{3} L^{2}\right) \lambda_{1} p^{2}+n \lambda_{1}=0,
$$

which gives

$$
n=-4 L^{2}\left(A k^{3}+B m^{3}\right) .
$$

Lastly, the bright soliton solution for space-time fractional coupled NNV equation is given by 


$$
u(x, y, t)=\lambda_{1} \operatorname{sech}^{2} \theta
$$

and

$$
v(x, y, t)=\lambda_{2} \operatorname{sech}^{2} \theta
$$

and

$$
w(x, y, t)=\lambda_{3} \operatorname{sech}^{2} \theta
$$

where the velocity of the solitons $n$ is given in (41), free parameters $\lambda_{1}, \lambda_{2}$ and $\lambda_{3}$ are given by (39), (34) and (35) respectively.

\section{Description of the $\left(\frac{G^{\prime}}{G}\right)$ expansion method for solving FDEs}

Suppose that the solution of ODE (8) can be expressed by a polynomial in $\left(G^{\prime} / G\right)$ as:

$$
U=\sum_{i=0}^{z} a_{i}\left(\frac{G^{\prime}}{G}\right)^{i}, \quad a_{z} \neq 0,
$$

where $G=G(\xi)$ satisfies the second order LODE in the form [71]

$$
\frac{d^{2} G(\xi)}{d \xi^{2}}+\lambda \frac{d G(\xi)}{d \xi}+\mu G(\xi)=0
$$

where $a_{1}, \ldots, a_{z}, \lambda$ and $\mu$ are constants will be determined later, $z$ is the positive integer which can be determined by the homogeneous balance with the highest order derivatives and highest order nonlinear appearing in ODE (8). When we substitute (45) into (8) and use Eq.(46), we collect all terms with the same order of $\left(G^{\prime} / G\right)$ together. When we equate all coefficient of this polynomial to zero, it gives us a set of algebraic equations for $a_{1}, \ldots, a_{z}, \lambda, \tau, \varepsilon$ and $\mu$ by using Maple. Then substituting $a_{1}, \ldots, a_{z}, \lambda, \mu, \varepsilon, \tau$ and general solutions of Eq. (46) into (8) we can get a variety of exact solutions of the FDEs (5).

\subsection{Applications of the proposed method}

\section{Example 1:}

In order to solve Eq.(11) by the $\left(G^{\prime} / G\right)$-expansion method, we use the traveling wave transformation (12) and with a similar approach in section 2, we get

$$
(k v-c) U+\frac{a k}{2} U^{2}+\tau c k^{2} L^{2} U^{\prime \prime}+\xi_{0}=0,
$$

where " $U^{\prime \prime}=\frac{d U}{d \xi}$ and $\xi_{0}$ is an integral constant. Balancing $U^{\prime \prime}$ with $U^{2}$ in (47) gives

$$
\begin{gathered}
2 z=z+2, \\
z=2 .
\end{gathered}
$$

Assume that it is possible to express solution of (47) by a polynomial in $\left(\frac{G^{\prime}}{G}\right)$ as:

$$
U(\xi)=a_{0}+a_{1}\left(\frac{G^{\prime}}{G}\right)+a_{2}\left(\frac{G^{\prime}}{G}\right)^{2}, \quad a_{2} \neq 0 .
$$

By using Eq.(46), from Eq.(49) we have

$$
\begin{aligned}
U^{\prime \prime}(\xi) & =6 a_{2}\left(\frac{G^{\prime}}{G}\right)^{4}+\left(2 a_{1}+10 a_{2} \lambda\right)\left(\frac{G^{\prime}}{G}\right)^{3} \\
& +\left(8 a_{2} \mu+3 a_{1} \lambda+4 a_{2} \lambda^{2}\right)\left(\frac{G^{\prime}}{G}\right)^{2} \\
& +\left(6 a_{2} \lambda \mu+2 a_{1} \mu+a_{1} \lambda^{2}\right)\left(\frac{G^{\prime}}{G}\right) \\
& +2 a_{2} \mu^{2}+a_{1} \lambda \mu .
\end{aligned}
$$

When we substitute Eqs.(49) and (50) into Eq.(47), collecting the coefficients of $\left(\frac{G^{\prime}}{G}\right)^{i}(i=$ $0, \ldots, 4)$ and set them to zero we get a system. The solutions of this algebraic equations can be done by Maple which gives

$$
\begin{aligned}
& a_{0}=\frac{c-v k-\tau c k^{2} L^{2} \lambda^{2}-8 \tau c k^{2} L^{2} \mu}{a k}, \\
& a_{1}=-\frac{12 \lambda \tau c k L^{2}}{a}, \\
& a_{2}=-\frac{12 \tau c k L^{2}}{a}, \\
& \xi_{0}=\frac{c^{2} k^{4} \tau^{2} L^{4}\left(8 \lambda^{2} \mu-16 \mu^{2}-\lambda^{4}\right)+c^{2}-2 v k c+v^{2} k^{2}}{2 a k} .
\end{aligned}
$$

where $\lambda$ and $\mu$ are arbitrary constants. By using Eq.(51), expression (49) can be written as

$$
\begin{aligned}
U(\xi)= & \frac{c-v k-\tau c k^{2} L^{2} \lambda^{2}-8 \tau c k^{2} L^{2} \mu}{a k} \\
& -\frac{12 \lambda \tau c k L^{2}}{a}\left(\frac{G^{\prime}}{G}\right)-\frac{12 \tau c k L^{2}}{a}\left(\frac{G^{\prime}}{G}\right)^{2} .
\end{aligned}
$$

When we substitute general solutions of Eq. (46) into Eq.(52) we have below travelling wave solutions of the equation as follows:

When $\lambda^{2}-4 \mu>0$, 


$$
\begin{array}{r}
U_{1}(\xi)=\frac{c-v k+2 \tau c k^{2} L^{2}\left(\lambda^{2}-4 \mu\right)}{a k}-\frac{3 \tau c k^{2} L^{2}\left(\lambda^{2}-4 \mu\right)}{a k} \times \\
\left(\frac{C_{1} \sinh \frac{1}{2} \sqrt{\lambda^{2}-4 \mu} \xi+C_{2} \cosh \frac{1}{2} \sqrt{\lambda^{2}-4 \mu} \xi}{C_{1} \cosh \frac{1}{2} \sqrt{\lambda^{2}-4 \mu} \xi+C_{2} \sinh \frac{1}{2} \sqrt{\lambda^{2}-4 \mu} \xi}\right)^{2},
\end{array}
$$

where $\xi=\frac{k x^{\alpha}}{\Gamma(1+\alpha)}-\frac{c t^{\alpha}}{\Gamma(1+\alpha)}$.

When $\lambda^{2}-4 \mu<0$,

$$
\begin{aligned}
U_{2}(\xi)= & \frac{c-v k+2 \tau c k^{2} L^{2}\left(\lambda^{2}-4 \mu\right)}{a k}+\frac{3 \tau c k^{2} L^{2}\left(\lambda^{2}-4 \mu\right)}{a k} \\
& \left(\frac{-C_{1} \sin \frac{1}{2} \sqrt{4 \mu-\lambda^{2}} \xi+C_{2} \cos \frac{1}{2} \sqrt{4 \mu-\lambda^{2}} \xi}{C_{1} \cos \frac{1}{2} \sqrt{4 \mu-\lambda^{2}} \xi+C_{2} \sin \frac{1}{2} \sqrt{4 \mu-\lambda^{2}} \xi}\right)^{2},
\end{aligned}
$$

where $\xi=\frac{k x^{\alpha}}{\Gamma(1+\alpha)}-\frac{c t^{\alpha}}{\Gamma(1+\alpha)}$.

In particular, if $C_{1} \neq 0, C_{2}=0, \lambda>0, \mu=0$, then $U_{1}$ and $U_{2}$ become

$$
\begin{aligned}
& u_{1}(x, t)=\frac{c-v k+2 \tau c k^{2} L^{2} \lambda^{2}}{a k} \\
& \quad+\frac{3 \tau c k L^{2} \lambda^{2}}{a} \operatorname{sech}^{2}\left(\frac{\lambda k x^{\alpha}}{2 \Gamma(1+\alpha)}-\frac{\lambda c t^{\alpha}}{2 \Gamma(1+\alpha)}\right) .
\end{aligned}
$$

When $\lambda^{2}-4 \mu=0$, we obtain rational function solution of Eq. (45)

$$
u_{2}(x, t)=\frac{c-v k}{a k}-\frac{12 \tau c k L^{2}}{a}\left(\frac{C_{1}}{C_{1}\left(\frac{k x^{\alpha}-c t^{\alpha}}{\Gamma(1+\alpha)}\right)+C_{2}}\right)^{2} .
$$

\section{Example 2:}

Similarly, in order to solve Eq. (24) by the proposed method, suppose that the solutions of the Eq. (26) can be expressed by a polynomial in $\left(\frac{G^{\prime}}{G}\right)$ as follows:

$$
\begin{aligned}
& U(\xi)=\sum_{i=0}^{z} a_{i}\left(\frac{G^{\prime}}{G}\right)^{i}, \quad a_{z} \neq 0, \\
& V(\xi)=\sum_{i=0}^{r} b_{i}\left(\frac{G^{\prime}}{G}\right)^{i}, \quad b_{r} \neq 0, \\
& W(\xi)=\sum_{i=0}^{p} c_{i}\left(\frac{G^{\prime}}{G}\right)^{i}, \quad c_{p} \neq 0 .
\end{aligned}
$$

By the same procedure as illustrated in example 1 , the homogeneous balance between highest order derivatives and non-linear terms in (26) we get positive integers $z=2, r=2$ and $p=2$. Consequently, we have:

$$
U(\xi)=a_{0}+a_{1}\left(\frac{G^{\prime}}{G}\right)+a_{2}\left(\frac{G^{\prime}}{G}\right)^{2}, a_{2} \neq 0,
$$

$$
V(\xi)=b_{0}+b_{1}\left(\frac{G^{\prime}}{G}\right)+b_{2}\left(\frac{G^{\prime}}{G}\right)^{2}, b_{2} \neq 0
$$

$$
W(\xi)=c_{0}+c_{1}\left(\frac{G^{\prime}}{G}\right)+c_{2}\left(\frac{G^{\prime}}{G}\right)^{2}, c_{2} \neq 0 .
$$

When we substitute Eqs. (50), (60)-(62) into Eq.(26), collecting the coefficients of $\left(\frac{G^{\prime}}{G}\right)^{i}(i=$ $0, \ldots, 3)$ and apply the same procedure of example 1 , we have

\section{Case 1:}

$$
\begin{aligned}
& a_{0}=a_{2} \mu, \quad a_{1}=a_{2} \lambda, \quad a_{2}=a_{2}, \\
& b_{0}=\frac{a_{2}^{2} \mu}{2 m^{2} L^{2}}, \quad b_{1}=\frac{a_{2}^{2} \lambda}{2 m^{2} L^{2}}, \quad b_{2}=\frac{a_{2}^{2}}{2 m^{2} L^{2}}, \\
& c_{0}=2 m^{2} L^{2} \mu, \quad c_{1}=2 m^{2} L^{2} \lambda, \quad c_{2}=2 m^{2} L^{2}, \\
& k=\frac{a_{2}}{2 m L^{2}}, \quad n=\frac{\left(4 \mu-\lambda^{2}\right)\left(A a_{2}^{3}+8 B m^{6} L^{6}\right)}{8 m^{3} L^{4}},
\end{aligned}
$$

where $\lambda$ and $\mu$ are arbitrary constants. Substituting Eq. (63) into Eqs.(60)-(62) yields

$$
U(\xi)=a_{2} \mu+a_{2} \lambda\left(\frac{G^{\prime}}{G}\right)+a_{2}\left(\frac{G^{\prime}}{G}\right)^{2}
$$

$$
V(\xi)=\frac{a_{2}^{2} \mu}{2 m^{2} L^{2}}+\frac{a_{2}^{2} \lambda}{2 m^{2} L^{2}}\left(\frac{G^{\prime}}{G}\right)+\frac{a_{2}^{2}}{2 m^{2} L^{2}}\left(\frac{G^{\prime}}{G}\right)^{2},
$$

$$
W(\xi)=2 m^{2} L^{2} \mu+2 m^{2} L^{2} \lambda\left(\frac{G^{\prime}}{G}\right)+2 m^{2} L^{2}\left(\frac{G^{\prime}}{G}\right)^{2}
$$

Then, when we substitute general solutions of Eq.(46) into Eqs.(64)-(66), we have two types of solutions of the Eqs.(24) as follows:

When $\lambda^{2}-4 \mu>0$, 


$$
\begin{aligned}
U_{1}(\xi) & =\frac{a_{2}\left(4 \mu-\lambda^{2}\right)}{4}\left(1-\Omega_{1}^{2}\right), \\
V_{1}(\xi) & =\frac{a_{2}^{2}\left(4 \mu-\lambda^{2}\right)}{8 m^{2} L^{2}}\left(1-\Omega_{1}^{2}\right), \\
W_{1}(\xi) & =\frac{m^{2} L^{2}\left(4 \mu-\lambda^{2}\right)}{2}\left(1-\Omega_{1}^{2}\right)
\end{aligned}
$$

where

$$
\begin{aligned}
& \Omega_{1}=\frac{K_{1} \sinh \frac{1}{2} \sqrt{\lambda^{2}-4 \mu} \xi+K_{2} \cosh \frac{1}{2} \sqrt{\lambda^{2}-4 \mu} \xi}{K_{1} \cosh \frac{1}{2} \sqrt{\lambda^{2}-4 \mu} \xi+K_{2} \sinh \frac{1}{2} \sqrt{\lambda^{2}-4 \mu} \xi}, \\
& \xi=\frac{a_{2} x^{\alpha}}{2 m L^{2} \Gamma(1+\alpha)}+\frac{m y^{\alpha}}{\Gamma(1+\alpha)}-\frac{\left(4 \mu-\lambda^{2}\right)\left(A a_{2}^{3}+8 B m^{6} L^{6}\right) t^{\alpha}}{8 m^{3} L^{4} \Gamma(1+\alpha)}
\end{aligned}
$$

When $\lambda^{2}-4 \mu<0$,

$$
\begin{aligned}
U_{2}(\xi) & =\frac{a_{2}\left(4 \mu-\lambda^{2}\right)}{4}\left(1+\Omega_{2}^{2}\right), \\
V_{2}(\xi) & =\frac{a_{2}^{2}\left(4 \mu-\lambda^{2}\right)}{8 m^{2} L^{2}}\left(1+\Omega_{2}^{2}\right), \\
W_{2}(\xi) & =\frac{m^{2} L^{2}\left(4 \mu-\lambda^{2}\right)}{2}\left(1+\Omega_{2}^{2}\right)
\end{aligned}
$$

where

$$
\Omega_{2}=\frac{-K_{1} \sin \frac{1}{2} \sqrt{4 \mu-\lambda^{2}} \xi+K_{2} \cos \frac{1}{2} \sqrt{4 \mu-\lambda^{2}} \xi}{K_{1} \cos \frac{1}{2} \sqrt{4 \mu-\lambda^{2}} \xi+K_{2} \sin \frac{1}{2} \sqrt{4 \mu-\lambda^{2}} \xi}
$$

In particular, if $K_{1} \neq 0, K_{2}=0, \mu=0$ then $U_{1}(\xi), V_{1}(\xi)$ and $W_{1}(\xi)$ become

$$
\begin{gathered}
u_{1}(x, y, t)=-\frac{\lambda^{2} a_{2}}{4} \operatorname{sech}^{2}(\Phi), \\
v_{1}(x, y, t)=-\frac{\lambda^{2} a_{2}^{2}}{8 m^{2} L^{2}} \operatorname{sech}^{2}(\Phi), \\
w_{1}(x, y, t)=-\frac{\lambda^{2} m^{2} L^{2}}{2} \operatorname{sech}^{2}(\Phi),
\end{gathered}
$$

where

$$
\Phi=\frac{\lambda a_{2} x^{\alpha}}{4 m L^{2} \Gamma(1+\alpha)}+\frac{\lambda m y^{\alpha}}{2 \Gamma(1+\alpha)}+\frac{\lambda^{3}\left(A a_{2}^{3}+8 B m^{6} L^{6}\right) t^{\alpha}}{16 m^{3} L^{4} \Gamma(1+\alpha)}
$$

Also if $K_{1} \neq 0, K_{2}=0, \mu=0$ then $U_{2}(\xi)$, $V_{2}(\xi)$ and $W_{2}(\xi)$ become, $u_{1}(x, y, t), v_{1}(x, y, t)$ and $w_{1}(x, y, t)$.

\section{Case 2:}

$$
\begin{aligned}
& a_{0}=\frac{a_{2}\left(2 \mu+\lambda^{2}\right)}{6}, \quad a_{1}=a_{2} \lambda, \quad a_{2}=a_{2}, \\
& b_{0}=\frac{a_{2}^{2}\left(2 \mu+\lambda^{2}\right)}{12 m^{2} L^{2}}, \quad b_{1}=\frac{a_{2}^{2} \lambda}{2 m^{2} L^{2}}, \quad b_{2}=\frac{a_{2}^{2}}{2 m^{2} L^{2}}, \\
& c_{0}=\frac{2 m^{2} L^{2} \mu+m^{2} L^{2} \lambda^{2}}{3}, c_{1}=2 m^{2} L^{2} \lambda, c_{2}=2 m^{2} L^{2}, \\
& k=\frac{a_{2}}{2 m L^{2}}, \quad n=\frac{\left(4 \mu-\lambda^{2}\right)\left(A a_{2}^{3}+8 B m^{6} L^{6}\right)}{8 m^{3} L^{4}},
\end{aligned}
$$

where $\lambda$ and $\mu$ are arbitrary constants. Substituting Eq.(76) into Eqs.(60)-(62), yields

$$
\begin{aligned}
& U(\xi)=\frac{a_{2}\left(2 \mu+\lambda^{2}\right)}{6}+a_{2} \lambda\left(\frac{G^{\prime}}{G}\right)+a_{2}\left(\frac{G^{\prime}}{G}\right)^{2}, \\
& V(\xi)=\frac{a_{2}^{2}}{2 m^{2} L^{2}}\left(\frac{\left(2 \mu+\lambda^{2}\right)}{6}+\lambda\left(\frac{G^{\prime}}{G}\right)+\left(\frac{G^{\prime}}{G}\right)^{2}\right) \\
& W(\xi)=m^{2} L^{2}\left(\frac{2 \mu+\lambda^{2}}{3}+2 \lambda\left(\frac{G^{\prime}}{G}\right)+2\left(\frac{G^{\prime}}{G}\right)^{2}\right) .
\end{aligned}
$$

When we substitute general solutions of Eq.(46) into Eqs.(77)-(79), we deduce the following traveling wave solutions:

When $\lambda^{2}-4 \mu>0$,

$$
\begin{gathered}
U_{3}(\xi)=\frac{a_{2}\left(4 \mu-\lambda^{2}\right)}{4}\left(\frac{1}{3}-\Omega_{1}^{2}\right), \\
V_{3}(\xi)=\frac{a_{2}^{2}\left(4 \mu-\lambda^{2}\right)}{8 m^{2} L^{2}}\left(\frac{1}{3}-\Omega_{1}^{2}\right), \\
W_{3}(\xi)=\frac{m^{2} L^{2}\left(4 \mu-\lambda^{2}\right)}{2}\left(\frac{1}{3}-\Omega_{1}^{2}\right) .
\end{gathered}
$$

When $\lambda^{2}-4 \mu<0$,

$$
\begin{gathered}
U_{4}(\xi)=\frac{a_{2}\left(4 \mu-\lambda^{2}\right)}{4}\left(\frac{1}{3}+\Omega_{2}^{2}\right), \\
V_{4}(\xi)=\frac{a_{2}^{2}\left(4 \mu-\lambda^{2}\right)}{8 m^{2} L^{2}}\left(\frac{1}{3}+\Omega_{2}^{2}\right), \\
W_{4}(\xi)=\frac{m^{2} L^{2}\left(4 \mu-\lambda^{2}\right)}{2}\left(\frac{1}{3}+\Omega_{2}^{2}\right) .
\end{gathered}
$$


In particular, if $K_{1} \neq 0, K_{2}=0, \mu=0$ then $U_{3}(\xi), V_{3}(\xi)$ and $W_{3}(\xi)$ become

$$
\begin{gathered}
u_{2}(x, y, t)=-\frac{\lambda^{2} a_{2}}{4}\left(\frac{1}{3}-\tanh ^{2}(\Phi)\right) \\
v_{2}(x, y, t)=-\frac{a_{2}^{2} \lambda^{2}}{8 m^{2} L^{2}}\left(\frac{1}{3}-\tanh ^{2}(\Phi)\right) \\
w_{2}(x, y, t)=-\frac{m^{2} L^{2} \lambda^{2}}{2}\left(\frac{1}{3}-\tanh ^{2}(\Phi)\right)
\end{gathered}
$$

Also if $K_{1} \neq 0, K_{2}=0, \mu=0$ then $U_{4}(\xi)$, $V_{4}(\xi)$ and $W_{4}(\xi)$ become, $u_{2}(x, y, t), v_{2}(x, y, t)$ and $w_{2}(x, y, t)$.

\section{Conclusion}

The ansatz and the $\left(G^{\prime} / G\right)$ expansion methods are used in this article to obtain some new exact solutions of the fractional regularized longwave equation and the fractional coupled NizhnikNovikov-Veselov equation. The $\left(G^{\prime} / G\right)$ expansion method is more effective and more general than the ansatz method because it gives exact solutions in more general forms. These methods are quite proficient methods for obtaining new exact solutions of FDEs. The obtained solutions are new and the methods can be extended to solve problems of nonlinear FDEs arising in the theory of solitons and other areas. To our knowledge, these new solutions have not been reported in former literature.

\section{References}

[1] Miller, K. S. and Ross, B. (1993). An Introduction to the Fractional Calculus and Fractional Differential Equations, Wiley, New York.

[2] Podlubny, I. (1999). Fractional Differential Equations, Academic Press, California.

[3] Kilbas, A. A., Srivastava, H. M. and Trujillo, J. J. (2006). Theory and Applications of Fractional Differential Equations, Elsevier, Amsterdam .

[4] Song, L., Wang, W. (2013). A new improved Adomian decomposition method and its application to fractional differential equations. Applied Mathematical Modelling. 37 (3) 1590-1598.

[5] Wang, Q. (2007). Homotopy perturbation method for fractional KdV equation. Appl. Math. Comput.. 190 1795-802

[6] Wu, G.C. and Baleanu, D. (2013). Variational iteration method for fractional calculus - a universal approach by Laplace transform. Advances in Difference Equations. 201318.
[7] Cui, M. (2009). Compact finite difference method for the fractional diffusion equation. Journal of Computational Physics. 228 (20) 7792-7804.

[8] Khan, N.A., Ara, A. and Mahmood, A. (2012). Numerical solutions of time-fractional Burgers equations: a comparison between generalized differential transformation technique and homotopy perturbation method. Int. J. Num. Meth. Heat \& Fl. Flow. 22 (2) 175-193.

[9] Song, L. and Zhang, H. (2007). Application of homotopy analysis method to fractional KdV-BurgersKuramoto equation. Phys. Lett. A. 367 88-94.

[10] El-Ajou, A., Odibat, Z., Momani, S. and Alawneh, A. (2010). Construction of analytical solutions to fractional differential equations using homotopy analysis method. Int. J. Appl. Math. 40 (2) 43-51.

[11] Tian, S.F. and Zhang, H.Q. (2012). On the integrability of a generalized variable-coefficient KadomtsevPetviashvili equation. Journal of Physics A: Mathematical and Theoretical. 45, 055203.

[12] Tian, S.F. and Zhang, H.Q. (2014). On the integrability of a generalized variable-coefficient forced Korteweg-de Vries equation in fluids. Stud. Appl. Math.132, 212-246.

[13] Mohyud-Din, S., Yıldırım, A. and Yülüklü, E. (2012). Homotopy analysis method for space- and timefractional $\mathrm{KdV}$ equation, Int. J. Num. Meth. Heat 8 Fl. Flow. 22 (7), 928-941.

[14] Sahoo, S. and Ray, S.S. (2015). Improved fractional sub-equation method for $(3+1)$-dimensional generalized fractional $\mathrm{KdV}$-Zakharov-Kuznetsov equations. Computers and Mathematics with Applications. 70, 158-166.

[15] Zhang, S. and Zhang, H.Q. (2011). Fractional subequation method and its applications to nonlinear fractional PDEs. Phys. Lett. A. 375, 1069-1073.

[16] Tian, S.F. (2017). Initial-boundary value problems for the general coupled nonlinear Schrödinger equation on the interval via the Fokas method. J. Differential Equations, 262, 506-558.

[17] Tian, S.F. (2016). The mixed coupled nonlinear Schrödinger equation on the half-line via the Fokas method. Proc. R. Soc. Lond. A. 472, 20160588.

[18] Bekir, A., Guner, O. and Unsal, O. (2015). The first integral method for exact solutions of nonlinear fractional differential equations, J. Comput. Nonlinear Dynam. 10(2), 021020-5.

[19] Baleanu, D., Uğurlu, Y. and Kilic, B. (2015). Improved $(G / / G)$ - expansion method for the timefractional biological population model and CahnHilliard equation. J. Comput. Nonlinear Dynam. 10 (5) 051016.

[20] Tu, J.M., Tian, S.F., Xu, M.J., Ma, P.L. and Zhang, T.T. (2016). On periodic wave solutions with asymptotic behaviors to a (3+1)-dimensional generalized B-type Kadomtsev-Petviashvili equation in fluid dynamics. Comput. E Math. Appl. 72, 2486-2504.

[21] Bekir, A., Guner, O., Bhrawy, A.H. and Biswas, A. (2015). Solving nonlinear fractional differential equations using exp-function and $\left(G^{\prime} / G\right)$-expansion methods. Rom. Journ. Phys. 60(3-4), 360-378.

[22] Bulut, H., Baskonus, H M. and Pandir, Y. (2013). The modified trial equation method for fractional wave equation and time fractional generalized Burgers equation. Abstract and Applied Analysis. 636802. 
[23] Guner, O. and Eser, D. (2014). Exact solutions of the space time fractional symmetric regularized long wave equation using different methods. Advances in Mathematical Physics. 456804 .

[24] Zhang, S., Zong Q-A., Liu, D. and Gao, Q. (2010). A generalized exp-function method for fractional riccati differential equations. Communications in Fractional Calculus. 1, 48-51.

[25] Guner, O., Bekir, A. and Bilgil, H. (2015). A note on exp-function method combined with complex transform method applied to fractional differential equations. Adv. Nonlinear Anal. 4(3), 201-208.

[26] Kaplan, M., Bekir, A., Akbulut, A. and Aksoy, E. (2015). The modified simple equation method for nonlinear fractional differential equations. Rom. Journ. Phys. 60(9-10), 1374-1383.

[27] Kaplan, M., Akbulut, A. and Bekir, A. (2016). Solving space-time fractional differential equations by using modified simple equation method, Commun. Theor. Phys. 65(5), 563-568.

[28] Aksoy, E., Kaplan, M. and Bekir, A. (2016). Exponential rational function method for space-time fractional differential equations. Waves in Random and Complex Media. 26(2), 142-151.

[29] Guner, O. (2015). Singular and non-topological soliton solutions for nonlinear fractional differential equations. Chin. Phys. B. 24(10), 100201.

[30] Guner, O. and Bekir, A. (2016). Bright and dark soliton solutions for some nonlinear fractional differential equations. Chin. Phys. B. 25(3), 030203.

[31] Tu, J.M., Tian, S.F., Xu, M.J. and Zhang, T.T. (2016). Quasi-periodic waves and solitary waves to a generalized KdV-Caudrey-Dodd-Gibbon equation from fluid dynamics. Taiwanese J. Math. 20, 823-848.

[32] Tu, J.M., Tian, S.F., Xu, M.J. and Zhang, T.T. (2016). On Lie symmetries, optimal systems and explicit solutions to the Kudryashov-Sinelshchikov equation, Appl. Math. Comput. 275, 345-352.

[33] Lin, S.D. and Lu, C.H. (2013). Laplace transform for solving some families of fractional differential equations and its applications. Advances in Difference Equations. 137.

[34] Srivastava, H.M., Golmankhaneh, A.K., Baleanu, D. and Yang, X.J. (2014). Local fractional Sumudu transform with application to IVPs on Cantor sets. Abstract and Applied Analysis. 620529.

[35] Wang, X.B., Tian, S.F., Xua, M.J. and Zhang T.T. (2016). On integrability and quasi-periodic wave solutions to a $(3+1)$-dimensional generalized $\mathrm{KdV}$-like model equation. Appl. Math. Comput. 283, 216-233.

[36] Arnous, A.H. and Mirzazadeh, M. (2015). Bäcklund transformation of fractional Riccati equation and its applications to the space-time FDEs. Mathematical Methods in the Applied Sciences. 38(18), 4673-4678.

[37] Feng, L.L., Tian, S.F., Wang, X.B. and Zhang, T.T. (2017). Rogue waves, homoclinic breather waves and soliton waves for the $(2+1)$-dimensional B-type Kadomtsev-Petviashvili equation. Appl. Math. Lett. $65,90-97$.

[38] Tian, S.F., Zhang, Y.F., Feng, B.L. and Zhang, H.Q. (2015). On the Lie algebras, generalized symmetries and Darboux transformations of the fifth-order evolution equations in shallow water. Chin. Ann. Math. 36B, 543-560.
[39] Tian, S.F., Wang, Z. and Zhang, H.Q. (2010). Some types of solutions and generalized binary Darboux transformation for the mKP equation with selfconsistent sources. J. Math. Anal. Appl. 366, 646-662.

[40] Al-Shara, S. (2014). Fractional transformation method for constructing solitary wave solutions to some nonlinear fractional partial differential equations. Applied Mathematical Sciences. 8, 5751-5762.

[41] Xu, M.J., Tian, S.F., Tu, J.M. and Zhang T.T. (2016). Bäcklund transformation, infinite conservation laws and periodic wave solutions to a generalized $(2+1)$ dimensional Boussinesq equation. Nonlinear Anal.: Real World Appl. 31, 388-408.

[42] Li, Z.B. and He, J. H. (2011). Application of the fractional complex transform to fractional differential equations. Nonlinear Sci. Lett. A. 2121-126.

[43] Caputo, M. (1967). Linear models of dissipation whose $\mathrm{Q}$ is almost frequency independent II, Geophys. $J$. Royal Astronom. Soc. 13, 529-539.

[44] Jumarie, G. (2006). Modified Riemann-Liouville derivative and fractional Taylor series of nondifferentiable functions further results. Comput. Math. Appl. 51, 1367-1376.

[45] Jumarie, G. (2009). Table of some basic fractional calculus formulae derived from a modified RiemannLiouvillie derivative for nondifferentiable functions. Appl. Maths. Lett.. 22, 378-385.

[46] He, J H., Elegan, S.K. and Li, Z.B. (2012). Geometrical explanation of the fractional complex transform and derivative chain rule for fractional calculus. Phys. Lett. A. 376, 257-259.

[47] Biswas, A. (2008). 1-soliton solution of the $\mathrm{K}(\mathrm{m}, \mathrm{n})$ equation with generalized evolution. Phys. Lett. A. 372, 4601-4602.

[48] Triki, H., Wazwaz, A.M. (2009). Bright and dark soliton solutions for a $\mathrm{K}(\mathrm{m}, \mathrm{n})$ equation with t-dependent coefficients. Phys. Lett. A. 373, 2162-2165.

[49] Bekir, A., Guner, O. (2013). Bright and dark soliton solutions of the $(3+1)$-dimensional generalized Kadomtsev-Petviashvili equation and generalized Benjamin equation. Pramana J. Phys. 81, 203.

[50] Triki, H., Milovic, D. and Biswas, A. (2013). Solitary waves and shock waves of the KdV6 equation. Ocean Engineering. 73, 119-125.

[51] Younis, M. and Ali, S. (2015). Bright, dark, and singular solitons in magneto-electro-elastic circular rod. Waves in Random and Complex Media. 25(4), 549555.

[52] Bekir, A. and Guner, O. (2013). Topological (dark) soliton solutions for the Camassa-Holm type equations. Ocean Engineering. 74, 276-279.

[53] Abdel-Salam, E.A.B., Hassan, G.F. (2016). Solutions to class of linear and nonlinear fractional differential equations. Commun. Theor. Phys.. 65, 127-135.

[54] Peregrine, D.H. (1966). Calculations of the development of an undular bore. J. Fluid Mech. 25, 321-330.

[55] Peregrine, D.H. (1967). Long waves on a beach. J. Fluid Mech. 27, 815-827.

[56] Benjamin, T.B., Bona, J.L. and Mahony, J. (1972). Model equations for waves in nonlinear dispersive systems. J. Philos. Trans. R. Soc. Lond. 227, 47-78.

[57] Abdel-Salam, E.A.B., Yousif, E.A. (2013). Solution of nonlinear space-time fractional differential equations using the fractional riccati expansion method. Mathematical Problems in Engineering. 846283. 
[58] Esen, A. and Kutluay, S. (2006). Application of a lumped Galerkin method to the regularized long wave equation. Appl. Math. Comput. 174, 833-845.

[59] Dag, I. (2000). Least square quadratic B-spline finite element method for the regularized long wave equation. Comp. Meth. Appl. Mech. Eng. 182, 205-215.

[60] Dag, I. and Ozer, M.N. (2001). Approximation of RLW equation by least square cubic B-spline finite element method. Appl. Math. Model. 25, 221-231.

[61] Saka, B., Dag, I. and Irk, D. (2008). Quintic Bspline collocation method for numerical solutions of the RLW equation. Anziam J. 49(3), 389-410.

[62] Saka, B., Sahin, A., Dag, I. (2011). B-spline collocation algorithms for numerical solution of the RLW equation. Numer. Meth. Part. D. E. 27, 581-607.

[63] Yusufoglu, E. and Bekir, A. (2007). Application of the variational iteration method to the regularized long wave equation. Computers and Mathematics with Applications. 54, 1154-1161.

[64] Liu, Y. and Yan, L. (2013). Solutions of fractional Konopelchenko-Dubrovsky and Nizhnik-NovikovVeselov equations using a generalized fractional subequation method. Abstract and Applied Analysis. 839613.

[65] Hong, T., Wang, Y.Z. and Huo, Y.S. (1998). Bogoliubov quasiparticles carried by dark solitonic excitations in nonuniform Bose Einstein condensates. Chin. Phys. Lett. 15, 550552.

[66] Das, G.C. (1997). Explosion of soliton in a multicomponent plasma. Phys. Plasmas. 4, 2095-2100.
[67] Lou, S.Y. (1999). A direct perturbation method: Nonlinear Schrodinger equation with loss. Chin. Phys. Lett. 16, 659-661.

[68] Shin, B.C., Darvishi, M.T. and Barati, A. (2009). Some exact and new solutions of the Nizhnik Novikov Vesselov equation using the Exp-function method. Computers and Mathematics with Applications. 58, 2147-2151.

[69] Deng, C. (2010). New abundant exact solutions for the $(2+1)$-dimensional generalized Nizhnik-NovikovVeselov system. Commun Nonlinear Sci Numer Simulat. 15, 3349-3357

[70] Boubir, B., Triki, H. and Wazwaz, A.M. (2013). Bright solitons of the variants of the Novikov-Veselov equation with constant and variable coefficients. Applied Mathematical Modelling. 37, 420-431.

[71] Wang, M.L., Li X. and Zhang, J. (2008). The $\left(G^{\prime} / G\right)$-expansion method and travelling wave solutions of nonlinear evolution equations in mathematical physics. Phys. Lett. A. 372(4), 417-423.

Özkan Güner is currently an assistant professor at Cankiri Karatekin University. He obtained his Ph.D. degree from Eskisehir Osmangazi University. He has published more than 70 articles in ISI-listed journals with an h-index of 11 . His current research interest mainly covers in exact solution of nonlinear partial and fractional differential equations.

An International Journal of Optimization and Control: Theories \& Applications (http://ijocta.balikesir.edu.tr)

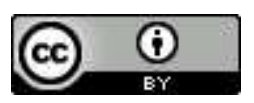

This work is licensed under a Creative Commons Attribution 4.0 International License. The authors retain ownership of the copyright for their article, but they allow anyone to download, reuse, reprint, modify, distribute, and/or copy articles in IJOCTA, so long as the original authors and source are credited. To see the complete license contents, please visit http://creativecommons.org/licenses/by/4.0/. 